\title{
Comparative Study of Astaxanthin, Cholesterol, Fatty Acid Profiles, and Quality Indices Between Shrimp Oil Extracted From Hepatopancreas and Cephalothorax
}

\author{
Navaneethan Raju ${ }^{1}$, Saqib Gulzar ${ }^{1}$, Natchaphol Buamard ${ }^{1}$, Lukai Ma ${ }^{2,3}$, Xiaoguo Ying ${ }^{4,5}$, \\ Bin Zhang $^{6}$ and Soottawat Benjakul ${ }^{1 *}$
}

\begin{abstract}
${ }^{1}$ International Center of Excellence in Seafood Science and Innovation, Faculty of Agro-Industry, Prince of Songkla University, Hat Yai, Thailand, ${ }^{2}$ Guangdong Provincial Key Laboratory of Lingnan Specialty Food Science and Technology, College of Light Industry and Food, Zhongkai University of Agriculture and Engineering, Guangzhou, China, ${ }^{3}$ Academy of Contemporary Agricultural Engineering Innovations, Zhongkai University of Agriculture and Engineering, Guangzhou, China, ${ }^{4}$ Zhejiang Provincial Key Laboratory of Health Risk Factors for Seafood, Collaborative Innovation Center of Seafood Deep Processing, College of Food and Pharmacy, Zhejiang Ocean University, Zhoushan, China, ${ }^{5}$ College of Biosystems Engineering and Food Science, Zhejiang University, Hangzhou, China, ${ }^{6}$ College of Food and Pharmacy, Zhejiang Ocean University, Zhoushan, China
\end{abstract}

\section{OPEN ACCESS}

Edited by:

Nilesh Prakash Nirmal, Mahidol University, Thailand

Reviewed by:

Yilmaz Uçar,

Çukurova University, Turkey

Ramesh Kumar Saini,

Konkuk University, South Korea

*Correspondence:

Soottawat Benjaku

soottawat.b@psu.ac.th

Specialty section: This article was submitted to

Food Chemistry,

a section of the journal

Frontiers in Nutrition

Received: 28 October 2021 Accepted: 10 November 2021 Published: 15 December 2021

Citation:

Raju N, Gulzar S, Buamard N, Ma L, Ying X, Zhang B and Benjakul S (2021)

Comparative Study of Astaxanthin, Cholesterol, Fatty Acid Profiles, and

Quality Indices Between Shrimp Oil

Extracted From Hepatopancreas and Cephalothorax. Front. Nutr. 8:803664.

doi: 10.3389/fnut.2021.803664
Shrimp oil from two different portions of Pacific white shrimp including cephalothorax and hepatopancreas was extracted using the mixture of hexane/isopropanol (1:1). The extracted oils from the cephalothorax (CPO) and hepatopancreas (HPO) were characterized for astaxanthin content, cholesterol levels, and fatty acid profiles. Nutrition indices of $\mathrm{CPO}$ and HPO were also compared. CPO had lower extraction yield (3.2 \pm $0.1 \%$, wet weight basis) than HPO (11.1 $\pm 0.5 \%$, wet weight basis). High-performance liquid chromatography results indicated that the astaxanthin content in HPO was higher, compared to that of CPO. Nevertheless, the cholesterol level in HPO was 70\% lower than that of CPO. Fatty acid profiles of HPO and CPO demonstrated that the polyunsaturated fatty acid (PUFA) content in HPO was higher than that of CPO. The amount of docosahexaenoic acid in the former was $\sim 2$ times higher than that of the latter. HPO contained $42.76 \pm 0.36 \%$ PUFA, whereas PUFA content of CPO was 35.27 $\pm 0.19 \%$. On the other hand, saturated fatty acids (SFA) were more pronounced in $\mathrm{CPO}(38.44 \pm 0.26 \%)$ than HPO (30.82 $\pm 0.55 \%)$. Based on nutrition indices, namely, atherogenicity index, thrombogenicity index, hypocholesterolemic/hypercholesterolemic $(\mathrm{h} / \mathrm{H})$ ratio, and PUFA/SFA ratio, HPO possessed higher health benefit than CPO. The oxidation status of CPO and HPO measured in terms of peroxide value, thiobarbituric acid reactive substances, anisidine value, and conjugated dienes indicated that higher primary oxidation products were present in CPO, whereas HPO exhibited more secondary oxidation compounds. Fourier transform infrared spectra further substantiated the presence of oxidation products in CPO and HPO. Liquid chromatography-mass spectrometry identification showed the enhanced levels of phospholipids and glycolipids in the ethanolic fraction of CPO. Overall, HPO with a higher yield was more beneficial in terms of health benefits than CPO.

Keywords: shrimp oil, nutrition indices, PUFA, astaxanthin, cholesterol 


\section{INTRODUCTION}

Increased shrimp demand, particularly in the forms of readyto-cook or ready-to-eat, has led to an increasing amount of farmed shrimp (1). During the processing, byproducts, namely, cephalothorax, carapace, tail, and internal organs were produced and discarded (2). Apart from whole shrimp or peeled shrimp, some products with cephalothorax free of hepatopancreas are in demand in some markets. Thus, the hepatopancreas is removed using the sucking machine (3). As a consequence, hepatopancreas rich in oil can serve as a potential source for oil extraction (3). The cephalothorax has been prominently utilized for producing chitin, protein hydrolysate, and shrimp oil (4, 5). Concerning shrimp oil production, hepatopancreas having higher lipid content seems to render higher yield, compared to cephalothorax (3). Takeungwongtrakul et al. (3) reported that hepatopancreas has $11.79 \pm 0.41 \%$ lipid content, while lipid content of $3.73 \pm 0.34 \%$ was found in cephalothorax. In addition, the residues after oil extraction have become less when hepatopancreas is used, compared to the cephalothorax. Shrimp oil is rich in astaxanthin, astaxanthin esters, and polyunsaturated fatty acids (PUFAs), especially eicosapentaenoic acid (EPA) and docosahexaenoic acid (DHA) $(6,7)$. These bioactive compounds are highly beneficial for improving human health. Astaxanthin, a powerful antioxidant, possesses antiaging, anti-inflammation, and anticancer properties $(5,8-10)$. DHA and EPA have been known for improving brain and cardiac health $(5,11,12)$. However, shrimp oil also contained saturated fatty acids (SFAs), which could lead to an increased low-density lipoprotein level and altered inflammatory response. In general, the limitation of SFAs in the diet is of concern about the ratio of unsaturated fatty acids (UFAs) to total fatty acids (13). Nutritive indices such as atherogenicity index (IA), thrombogenicity index (IT), hypocholesterolemic/hypercholesterolemic $(\mathrm{h} / \mathrm{H})$ ratio, and PUFA/SFA were computed for monitoring the quality of lipid (14). These indices could be employed for the shrimp oil to indicate nutritive value.

Although shrimp oil has a high content of highly beneficial bioactive compounds, it also contains cholesterol (6). Shrimp lipid extracted from cephalothorax consists of $65-70 \mathrm{mg}$ cholesterol/100 g of lipid (5-7). Despite the need for some cholesterol to make hormones, vitamin $\mathrm{D}$, etc., high levels of cholesterol can combine with other substances to form plaque in blood, causing atherosclerosis (15). To improve the nutrition quality of shrimp lipid extracted from cephalothorax, cholesterol has been lowered by saponin and beta-cyclodextrin (16-18). However, the cholesterol content in shrimp oil from the hepatopancreas has not been studied. The complete profiling of fatty acids, astaxanthin, cholesterol, and nutrition indices could be useful for the consumers to intake the shrimp oil for health benefits. To our knowledge, no aforementioned information for shrimp oils from both cephalothorax and hepatopancreas exists. Therefore, this study was aimed to characterize the oils extracted from cephalothorax and hepatopancreas of Pacific white shrimp and to compare both oils in terms of fatty acid content, astaxanthin content, cholesterol level, oxidation status, and nutritive indices.

\section{MATERIALS AND METHODS}

\section{Chemicals}

All the chemicals used in the experiment were of analytical grade and purchased from Merck (Darmstadt, Germany). Pacific white shrimp (Litopenaeus vannamei) cephalothorax (shrimp head) and hepatopancreas were gifted from Sea Wealth Frozen Food Co., Ltd., Songkhla province, Thailand and Sea Fresh Industry Public Company, Pak Nam, Mueang Chumphon, Thailand, respectively.

\section{Extraction of Oil From Cephalothorax and Hepatopancreas}

Shrimp oil was extracted using the hexane/isopropanol extraction method as detailed by Raju and Benjakul (6). First, cephalothorax and hepatopancreas were blended with a blender at high speed for $1 \mathrm{~min}$ to obtain a homogenous paste. The paste $(100 \mathrm{~g})$ was added to $500 \mathrm{ml}$ of hexane/isopropanol mixture (1:1). The mixture was homogenized at 9,000 rpm for 3 min with the aid of an IKA Labortechnik homogenizer (Selangor, Malaysia). After homogenization, the solvent phase was separated by centrifugation using a Hitachi centrifuge (Hitachi Koki Co., Ltd, Tokyo, Japan) at $3,000 \times g$ for $15 \mathrm{~min}$ at $4^{\circ} \mathrm{C}$. The collected solvent phase was washed with an equal volume of distilled water and the process was repeated thrice. The hexane phase was collected and anhydrous sodium sulfate $(10 \mathrm{~g})$ was added to remove the water traces, followed by filtering using a Whatman filter paper No. 4 (Whatman International Ltd., Maidstone, England). The solvent was evaporated using an EYELA rotary evaporator N-1000 (Tokyo Rikakikai, Co., Ltd., Tokyo, Japan) at $40^{\circ} \mathrm{C}$ to obtain shrimp oil. Shrimp oil from cephalothorax and hepatopancreas were termed as "CPO" and "HPO," respectively. Both the shrimp oils were flushed with nitrogen, placed in an amber vial, capped tightly, and stored at $-40^{\circ} \mathrm{C}$.

\section{Chemical Compositions, Nutritional, and Quality Indices of CPO and HPO}

Both CPO and HPO were subjected to analyses.

\section{Cholesterol and Astaxanthin Contents}

The cholesterol and astaxanthin contents were quantified by high-performance liquid chromatography (HPLC) (Waters 2695 series, Milford, MA, USA) $(6,19)$ equipped with a reversephase Thermo scientific BDS-C18 column $(5 \mu \mathrm{m} ; 150 \times 4 \mathrm{~mm})$ following the method of Raju et al. (20). Each oil sample $(100 \mu \mathrm{l})$ was dissolved in $1 \mathrm{ml}$ of ethanol. The mixture was vortexed vigorously for $1 \mathrm{~min}$ and stored at $-18^{\circ} \mathrm{C}$ for $2 \mathrm{~h}$. After incubation, the prepared mixtures were centrifuged at 3,600 $\times g$ for $10 \mathrm{~min}$. A $100 \mu \mathrm{l}$ of supernatant was taken and made up to $1 \mathrm{ml}$ using mobile phase and injected in HPLC. HPLC program was performed at isocratic condition using $1.2 \mathrm{ml} / \mathrm{min}$ flow rate of methanol-acetonitrile (50:50) mixture as the mobile phase. A photodiode-array detector (Waters 2998, Milford, MA, USA) was used for the detection at $480 \mathrm{~nm}$ for astaxanthin and $202 \mathrm{~nm}$ for cholesterol. Authentic standards of astaxanthin (Dr. Ehrenstorfer 
GmbH, Augsburg, Germany) and cholesterol (Acros organics, Morris Plains, NJ, USA) were used for identification. The content was expressed as $\mathrm{mg} / \mathrm{g}$ oil.

\section{Fatty Acid Composition}

Fatty acid profile was determined by the method of Raju et al. (20). Briefly, the sample $(10 \mathrm{mg})$ was dissolved in $1 \mathrm{ml}$ of hexane and esterified with $200 \mu \mathrm{l}$ of $2 \mathrm{M}$ methanolic sodium hydroxide at $50^{\circ} \mathrm{C}$ for $5 \mathrm{~min}$. After cooling down, the mixture was vortexed and $200 \mu \mathrm{l}$ of $2 \mathrm{M}$ methanolic hydrochloric acid was added. The prepared mixture was vortexed thoroughly and then centrifuged at $3,500 \times g$ for $10 \mathrm{~min}$. The hexane phase was collected and injected into gas chromatography (Agilent GC 7890B; Santa Clara, CA, USA). Injection temperature was maintained at $250^{\circ} \mathrm{C}$ and the initial column temperature was first reduced to $80^{\circ} \mathrm{C}$. The temperature was increased at $4^{\circ} \mathrm{C} \mathrm{min}{ }^{-1}$ for $40 \mathrm{~min}$ to $220^{\circ} \mathrm{C}$ and finally reached $240^{\circ} \mathrm{C}$. The eluted compounds were identified by a flame ionization detector (Agilent GC 7890B; Santa Clara, CA, USA) at $270^{\circ} \mathrm{C}$ as a detector temperature. Genuine standards (Supelco FAME mix, Bellefonte, PA, USA) were used for the peak identification and the fatty acid content was expressed as a percentage.

\section{Nutritional Indices}

Nutritional indices were calculated based on the fatty acid profile by the following formulas (14).

The index of atherogenicity $(\mathrm{IA})=[\mathrm{C} 12: 0+(4 \times \mathrm{C} 14: 0)+$ C16: 0] / [ $\Sigma$ MUFAs $+\Sigma$ PUFAn6 + PUFAn3]

The index of thrombogenicity $($ IT $)=[$ C14: $0+$ C16: $0+$ C18: $0] /[(0.5 \times \Sigma$ MUFA $)+(0.5 \times \Sigma \omega-6$ PUFA $)+(3 \times \Sigma \omega-3$

PUFA $)+(\omega-3 / \omega-6)]$

The hypocholesterolemic/hypercholesterolemic $(\mathrm{h} / \mathrm{H})$ ratio $=$ [cis C18: $1+\Sigma$ PUFA] [C12: $0+$ C14: $0+$ C16: 0]

PUFA/SFA ratio $=\Sigma$ PUFA $/ \Sigma$ SFA

\section{Lipid Oxidation}

Both samples were determined for lipid oxidation using different indices.

\section{Peroxide Value (PV)}

Peroxide value (PV) was determined by the method of Pudtikajorn and Benjakul (21). An oil sample (0.1 g) was added with $25 \mathrm{ml}$ of acetic acid/chloroform (3:2) mixture. To the prepared mixture, $1 \mathrm{ml}$ of saturated potassium iodide was added and mixed. The mixture was then incubated in dark for $5 \mathrm{~min}$. Subsequently, distilled water $(75 \mathrm{ml})$ was added and shaken. At last, to the prepared mixture, $0.5 \mathrm{ml}$ of $1 \%$ starch solution was added, shaken, and titrated with $0.01 \mathrm{~N}$ $\mathrm{Na}_{2} \mathrm{~S}_{2} \mathrm{O}_{3}$. The titration was stopped after the disappearance of the dark blue color. PV was calculated and reported as $\mathrm{mEq} /$ $\mathrm{kg}$ oil.

\section{Thiobarbituric Acid Reactive Substances (TBARS)}

Thiobarbituric acid reactive substances (TBARS) value was analyzed following the method of Gulzar and Benjakul (16). Quantification of the sample was done using the standard curve of 1,1,3,3-tetramethoxypropane (0-6 ppm) and the value was expressed as mg malonaldehyde/kg oil.

\section{p-Anisidine Value (AnV)}

$p$-Anisidine value $(\mathrm{AnV})$ was measured as tailored by Firestone (22). Briefly, an oil sample $(0.1 \mathrm{~g})$ was mixed with $25 \mathrm{ml}$ of isooctane and $0.5 \mathrm{ml}$ of $p$-anisidine reagent. The absorbance of the mixture was read at $350 \mathrm{~nm}$ using a UV/vis spectrophotometer (Shimadzu UV-1800, Kyoto, Japan). AnV was calculated as follows:

$$
A n V=25 \times \frac{(1.2 \times A 2)-A 1}{W}
$$

where $A 1$ and $A 2$ are the absorbances before and after adding $p$-anisidine, respectively; $W$ is the weight of the sample (g).

\section{Conjugated Diene}

Conjugated dienes (CDs) were analyzed using the method of Raju and Benjakul (17). Shrimp oil (0.1 g) was added to $100 \mathrm{ml}$ of isooctane. The absorbance of the solution was read at $234 \mathrm{~nm}$ $\left(A_{234}\right)$. CD was calculated using the following equation:

$$
C D=\frac{A_{234}}{\text { Weight of shrimp oil }(\mathrm{g}) \times \text { cell path length }(\mathrm{cm})}
$$

\section{Fourier Transform Infrared (FTIR) Spectra}

Fourier transform infrared (FTIR) spectra of samples were attained by Bruker Model Vector 33 FTIR spectrometer (Bruker Co., Ettlingen, Germany) following the method of Singh et al. (4). Wavenumber was selected between 4,000 and $500 \mathrm{~cm}^{-1}$ with 16 scans. A clean empty cell at $25^{\circ} \mathrm{C}$ was kept for normalization and as the reference background. The data were collected using OPUS 8.5 data collection software (Bruker Co. Billerica, MA, USA).

\section{LC-MS Identification of Ethanol-Soluble Lipids}

Ethanol soluble lipids were prepared using the method of Raju et al. (18). Briefly, oil samples $(100 \mathrm{mg})$ were added to $1 \mathrm{ml}$ of ethanol and vortexed vigorously. The prepared mixture was then stored at $-20^{\circ} \mathrm{C}$ for $3 \mathrm{~h}$. At the end of the incubation period, the mixtures were centrifuged at $4,000 \times g$ and the upper phase was collected. The upper phase $(500 \mu \mathrm{l})$ was subjected to liquid chromatography quadrupole time-of-flight mass spectrometer (LC-QTOF MS), 1290 Infinity II LC-6545 Quadrupole-TOF (Agilent Santa Clara, CA, USA). The instrument was equipped with Zorbax Eclipse Plus C18 column (150 mm length $\times 2.1 \mathrm{~mm}$ innerdiameter, particle size $1.8 \mu \mathrm{m}$ ) (Agilent Santa Clara, CA, USA). Mobile phase A: $50 \mathrm{mM}$ ammonium acetate/methanol [20/80 $(\mathrm{v} / \mathrm{v})]$ and B: Isopropanol/methanol [70/30 (v/v)] were used for the separation. Positive atmospheric pressure chemical ionization was done and compounds were identified based on mass and spectrum compared with MassHunter METLIN PCD library. 

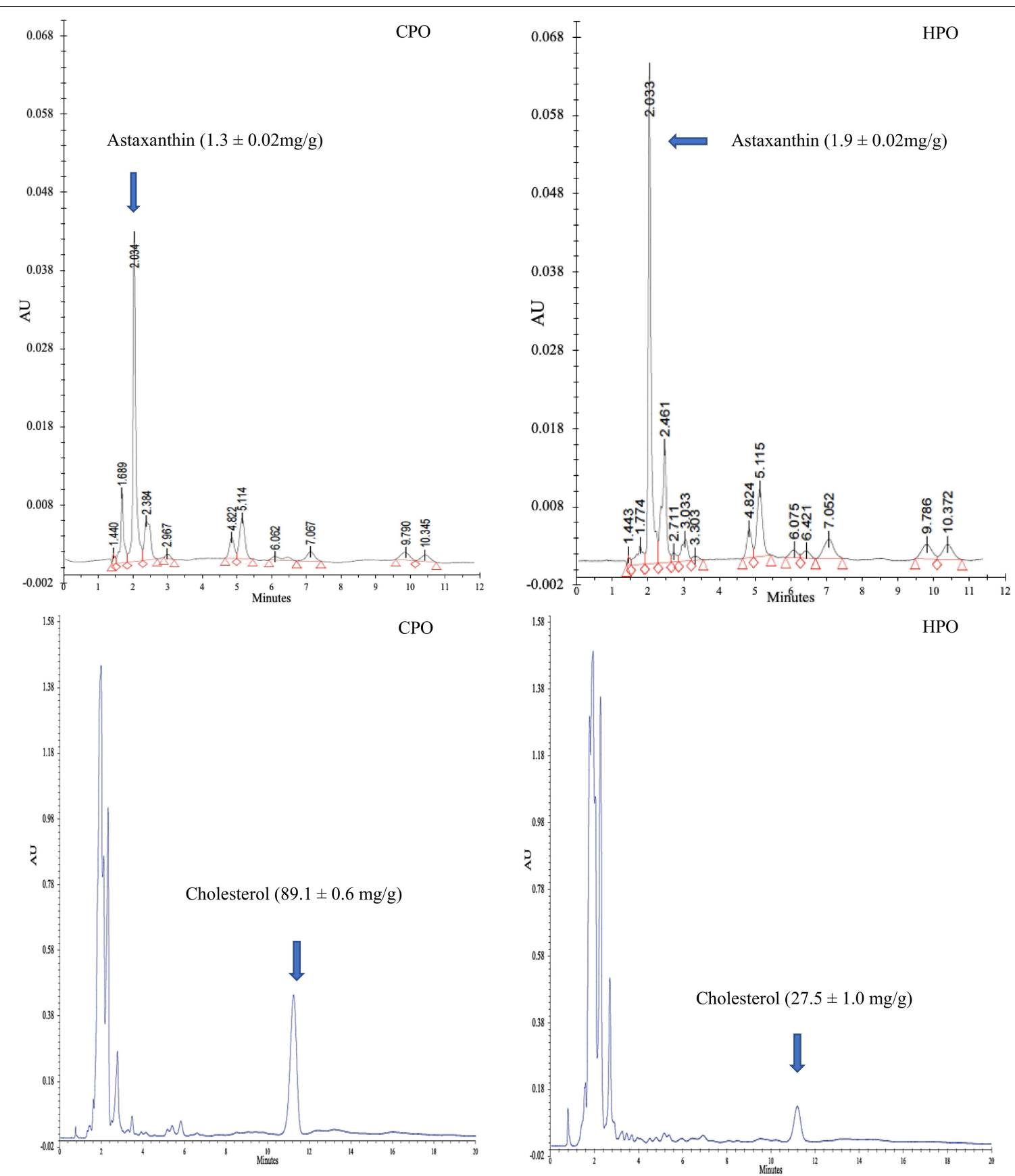

FIGURE 1 | HPLC chromatogram of astaxanthin and cholesterol from CPO and HPO. CPO: Oil extracted from cephalothorax; HPO: oil extracted from hepatopancreas.

\section{Statistical Analysis}

A completely randomized design was used for this study. Experiment and analysis were done in triplicate using three different sample lots. ANOVA was performed using the Statistical Package for Social Science SPSS software (IBM software, New York, NY, USA) (23). The $t$-test was used for pair comparison.

\section{RESULTS AND DISCUSSION}

\section{Chemical Compositions and Nutritional Indices of CPO and HPO}

$\mathrm{CPO}$ and $\mathrm{HPO}$ had different yields. Yields of $\mathrm{CPO}$ and $\mathrm{HPO}$ were $3.2 \pm 0.1 \%$ and $11.1 \pm 0.5 \%$ (wet weight basis), respectively. Takeungwongtrakul et al. (3) reported that cephalothorax of 
Pacific white shrimp had a fat content of $3.73 \%$ (wet weight basis), while hepatopancreas consisted of $11.79 \%$ fat. The cephalothorax is the fused head and thorax including the outer shell (carapace), internal organs, and other components (24). The hepatopancreas is an important organ, which plays a major role in digestion and absorption (25). During the production of whole shrimp free of hepatopancreas, hepatopancreas was removed directly by a vacuum sucking machine (26). As a result, the obtained hepatopancreas was not adulterated by other organs, shells, or components (26). Hepatopancreas also functions as a lipid depositing organ (25). This was witnessed by four times higher fat content than that of cephalothorax.

\section{Astaxanthin and Cholesterol}

Chromatograms of astaxanthin and cholesterol in HPO and CPO are shown in Figure 1. Peak height and peak area have been used to quantify the target compounds. Higher astaxanthin content but lower cholesterol content was found in HPO than CPO ( $p$ $<0.05$ ). Gómez-Estaca et al. (7) reported that oil from Pacific white shrimp cephalothorax had high cholesterol content (65 $\mathrm{mg} / \mathrm{g}$ ) but low astaxanthin content $(7 \mathrm{mg} / \mathrm{g})$. The hepatopancreas is a storage organ for carotenoids and lipids (25). As a principal carotenoid, astaxanthin plays a major role in photoprotection and as an antioxidant (27). For the biological response of crustaceans, astaxanthin is transported via hemolymph to encounter the response (28). As a primary carotenoid reservoir of the whole body (28), HPO contains high astaxanthin. However, as a principal sterol in crustaceans, cholesterol plays a vital role in maintaining cellular structure and controlling the rigidity of the membrane (29). Cholesterol maintains the membrane fluidity and protects crustaceans from salinity stress and temperature stress (29). Shrimps are bottom inhabitants and they survive in extreme environments (30). Cholesterol metabolism primarily takes place in the hepatopancreas (31) and is transported to the tissues for survivability and the control of stress (32) and to the ovaries for yolk development (31). Due to this reason, HPO contains lower cholesterol than CPO. In terms of health benefits, dietary cholesterol intake will lead to an increase in serum cholesterol levels and cardiovascular diseases (5). When comparing HPO and CPO, the former could provide more health benefits than the latter. Moreover, the augmented astaxanthin content would increase antioxidant activity, inflammatory protection, anticancer, and antiaging properties (5).

\section{Fatty Acid Profile}

Table 1 illustrates the fatty acid profile of CPO and HPO. Palmitic acid (SFA) was found to be the most dominant fatty acid in both oils. However, the second-highest fatty acid in HPO was DHA $(22: 6, n 3)$ at $16.25 \%$, which was higher than that of CPO $(8.98 \%)$. The second highest fatty acid in CPO was linoleic acid (18:2, n6 ), which constituted $14.22 \%$. On the other hand, n-6 fatty acid content was higher in the latter $(p<0.05)$. For oleic acid $(18: 1$, $n-9)$, CPO showed higher content (13.18\%) than HPO $(9.87 \%)$ $(p<0.05)$. The total SFA and MUFA contents were higher in CPO. However, the health-beneficial PUFA content was higher in HPO. In general, n-3 fatty acid content was greater in HPO
TABLE 1 | Fatty acid profiles of shrimp oil extracted from cephalothorax and hepatopancreas.

\begin{tabular}{|c|c|c|}
\hline $\begin{array}{l}\text { Fatty } \\
\text { acids (\%) }\end{array}$ & CPO & HPO \\
\hline C12:0 & $0.74 \pm 0.04 a$ & $0.31 \pm 0.01 b$ \\
\hline C14:0 & $0.48 \pm 0.01 b$ & $1.17 \pm 0.05 a$ \\
\hline C15:0 & $0.33 \pm 0.01 b$ & $0.80 \pm 0.01 a$ \\
\hline C15:1 & $0.35 \pm 0.01 a$ & ND \\
\hline C16:0 & $18.97 \pm 0.36 b$ & $19.27 \pm 0.61 a$ \\
\hline C16:1 & $1.10 \pm 0.01 b$ & $2.23 \pm 0.02 a$ \\
\hline C17:0 & $0.80 \pm 0.01 a$ & $0.45 \pm 0.04 b$ \\
\hline C17:1 & $0.50 \pm 0.00$ & ND \\
\hline C18:0 & $8.67 \pm 0.05 a$ & $1.95 \pm 0.04 b$ \\
\hline C18:1 n-9c & $13.18 \pm 0.17 a$ & $9.87 \pm 0.03 b$ \\
\hline C18:2 n-3c & $14.22 \pm 0.14 a$ & $13.13 \pm 0.02 b$ \\
\hline C20:0 & $0.46 \pm 0.01$ & ND \\
\hline C20:1 & $1.05 \pm 0.07 b$ & $1.77 \pm 0.14 a$ \\
\hline C18:3 n-3c & $0.43 \pm 0.01 b$ & $0.60 \pm 0.00 a$ \\
\hline C20:2 n-6c & $2.22 \pm 0.02 b$ & $2.85 \pm 0.04 a$ \\
\hline C20:0 & $0.76 \pm 0.02$ & $\mathrm{ND} \pm 0.00$ \\
\hline C23:0 & $6.42 \pm 0.03 b$ & $7.19 \pm 0.03 a$ \\
\hline C24:0 & $0.81 \pm 0.02$ & $\mathrm{ND} \pm 0.00$ \\
\hline C20:5 n-3c (EPA) & $9.42 \pm 0.02 b$ & $9.94 \pm 0.06 a$ \\
\hline C24:1 & $1.06 \pm 0.11$ & $\mathrm{ND} \pm 0.00$ \\
\hline C22:6 n-3c (DHA) & $8.98 \pm 0.03 b$ & $16.25 \pm 0.27 a$ \\
\hline Others & $9.06 \pm 0.12 b$ & $12.25 \pm 0.96 a$ \\
\hline $\begin{array}{l}\text { Saturated Fatty } \\
\text { Acid (SFA) }\end{array}$ & $38.44 \pm 0.26 a$ & $30.82 \pm 0.55 b$ \\
\hline $\begin{array}{l}\text { Monounsaturated } \\
\text { Fatty Acid (MUFA) }\end{array}$ & $17.24 \pm 0.01 a$ & $13.86 \pm 0.15 b$ \\
\hline $\begin{array}{l}\text { Polyunsaturated } \\
\text { Fatty Acid (PUFA) }\end{array}$ & $35.27 \pm 0.19 b$ & $42.76 \pm 0.36 a$ \\
\hline n-3 Fatty acids & $18.83 \pm 0.05 b$ & $26.79 \pm 0.32 a$ \\
\hline n-6 Fatty acids & $16.44 \pm 0.15 a$ & $15.98 \pm 0.05 b$ \\
\hline
\end{tabular}

CPO, Oil extracted from cephalothorax; HPO, oil extracted from hepatopancreas; EPA, eicosapentaenoic acid; DHA, docosahexaenoic acid.

Different lowercase letters within the same row denote significant difference $(p<0.05)$.

than CPO $(p<0.05)$. These results were in accordance with Gómez-Estaca et al. (7), Gulzar and Benjakul (16) who stated that palmitic acid was dominant in shrimp oil extracted from cephalothorax. Takeungwongtrakul et al. (3) documented that lipid from hepatopancreas contained lesser SFA content than that extracted from cephalothorax. Gómez-Estaca et al. (7) reported higher n-6 fatty acid than n-3 fatty acid in CPO from Pacific white shrimp. Increased n-6 fatty acid consumption has been known to produce eicosanoids that cause inflammation, while n3 fatty acids show anti-inflammatory activity (33). Overall HPO contained fatty acids with health benefits to a higher extent, compared to CPO, mainly due to the presence of n-3 fatty acids, especially EPA and DHA.

\section{Nutrition Indices}

Dietary oils extracted from plants and animals are composed of numerous fatty acids, classified as SFA, MUFA, and PUFA. 

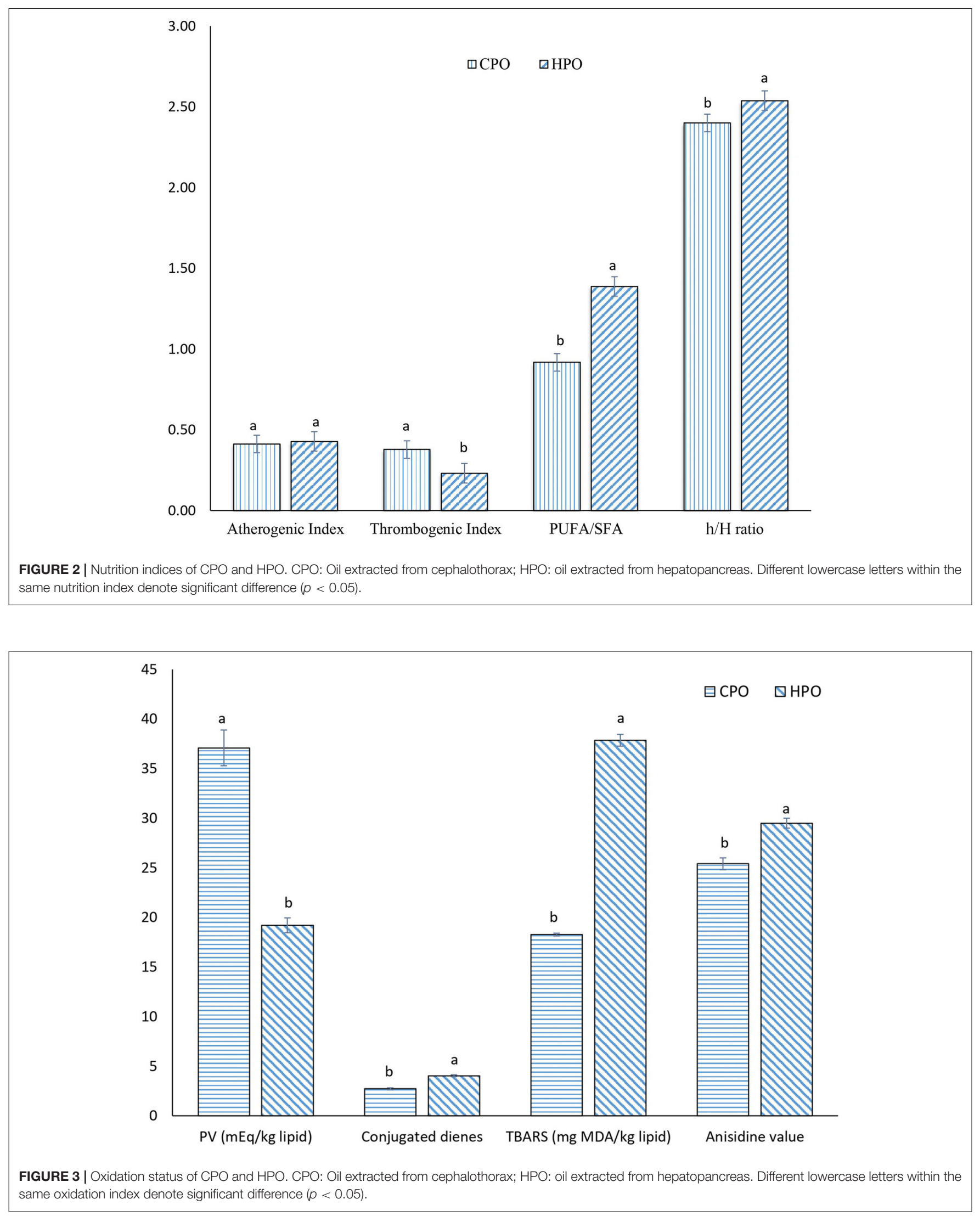


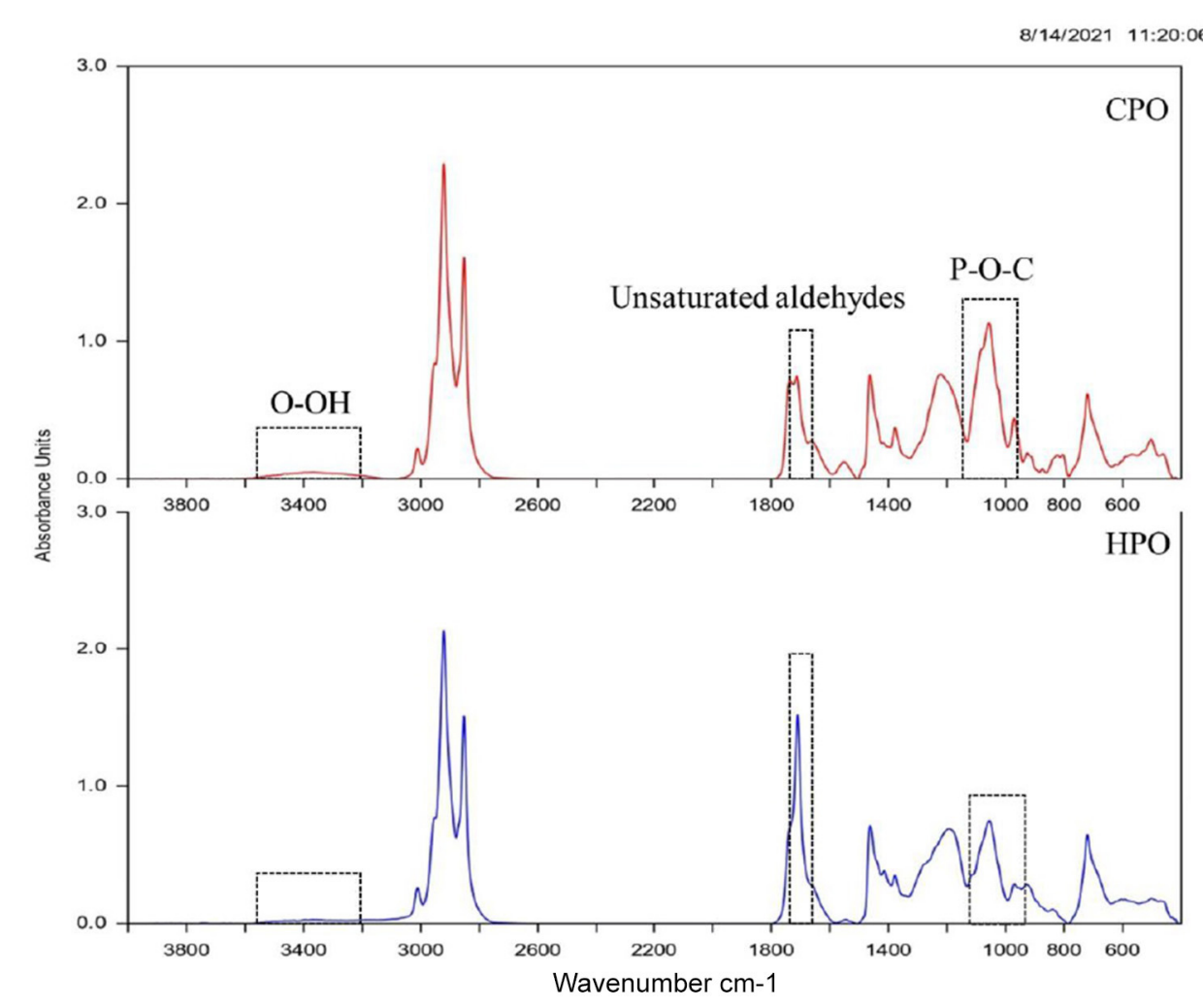

FIGURE 4 | FTIR spectra of CPO and HPO. CPO: Oil extracted from cephalothorax; HPO: oil extracted from hepatopancreas.

However, the health benefits of the extracted oil are governed by the presence of beneficial PUFA with a low ratio of SFA (14). From fatty acid composition, nutrition indices were calculated to reveal the health-promoting index. Commonly used nutritive indices are IA, IT, and $\mathrm{h} / \mathrm{H}$ (14). Apart from these, several indices such as PUFA/SFA, EPA + DHA, trans fatty acid, and unsaturation index have been also used to indicate the health benefit (13). In this current study, IA, IT, h/H, and PUFA/SFA were computed for both CPO and HPO (Figure 2).

A similar IA index was observed between CPO and HPO $(p>$ 0.05). However, IT was lower in HPO, compared to that of CPO. Ulbricht and Southgate (34) developed and proposed IA and IT for calculating atherogenicity and thrombogenicity $(14,34)$. Atherogenicity for foods is calculated based upon SFA and UFA contents (35). Lauric acid (C12: 0), myristic acid (C14: 0), and palmitic acid (C16: 0) are considered to be atherogenic (35). These fatty acids can bind with the cells of the circulatory system, leading to the formation of plague (14). However, UFAs possess antiatherogenic activity, thus inhibiting plaque formation. Thus, IA was calculated from the ratio of proatherogenic SFAs to antiatherogenic UFAs (14). Thrombogenicity refers to the ratio of fatty acids inducing clot formation (C12:0, C14:0, and C16:0) and the fatty acids having antithrombotic effects such as MUFA, n-6, and n-3 fatty acids (34). Since HPO contained a higher amount of n-3 fatty acids, compared to CPO, this resulted in the lower IT in HPO. IA and IT have been considered as markers for assessing cardiovascular health (35). When comparing both the oils, HPO was considered as the better oil in terms of protecting heart diseases with higher anticlotting ability.

The $\mathrm{h} / \mathrm{H}$ ratio was first developed in lamb meat by SantosSilva et al. (36). The $\mathrm{h} / \mathrm{H}$ ratio was found to be upgraded from PUFA/SFA ratio by selecting only three SFA (C12:0, C14: 0 , and $\mathrm{C} 16: 0$ ) that can induce hypercholesterolemic conditions rather than selecting total SFA. High $\mathrm{h} / \mathrm{H}$ ratio and PUFA/SFA ratio are considered as health benefits in neutralizing hypercholesterolemic conditions mainly by PUFA (35). From a nutritional point of view, the $\mathrm{h} / \mathrm{H}$ ratio between 0.5 and 1 is considered as an ideal value (37). According to the Dietary Guidelines for Americans and the European Society of Cardiology, the upper limit for the intake of total SFA is set at $7-10 \%$ of total energy intake, whereas the upper limit for consumption of PUFA is set between 6 and 11\% of total energy intake (38). HPO possessed a higher $\mathrm{h} / \mathrm{H}$ ratio and PUFA/SFA ratio. The augmented PUFA content in HPO led to a profound hypocholesterolemic effect as witnessed by an increased $\mathrm{h} / \mathrm{H}$ ratio.

\section{Lipid Oxidation}

Lipid oxidation was assayed by measuring primary and secondary lipid oxidation products (Figure 3). PV has been 
TABLE 2 | LC-MS identification of shrimp oil extracted from cephalothorax and hepatopancreas.

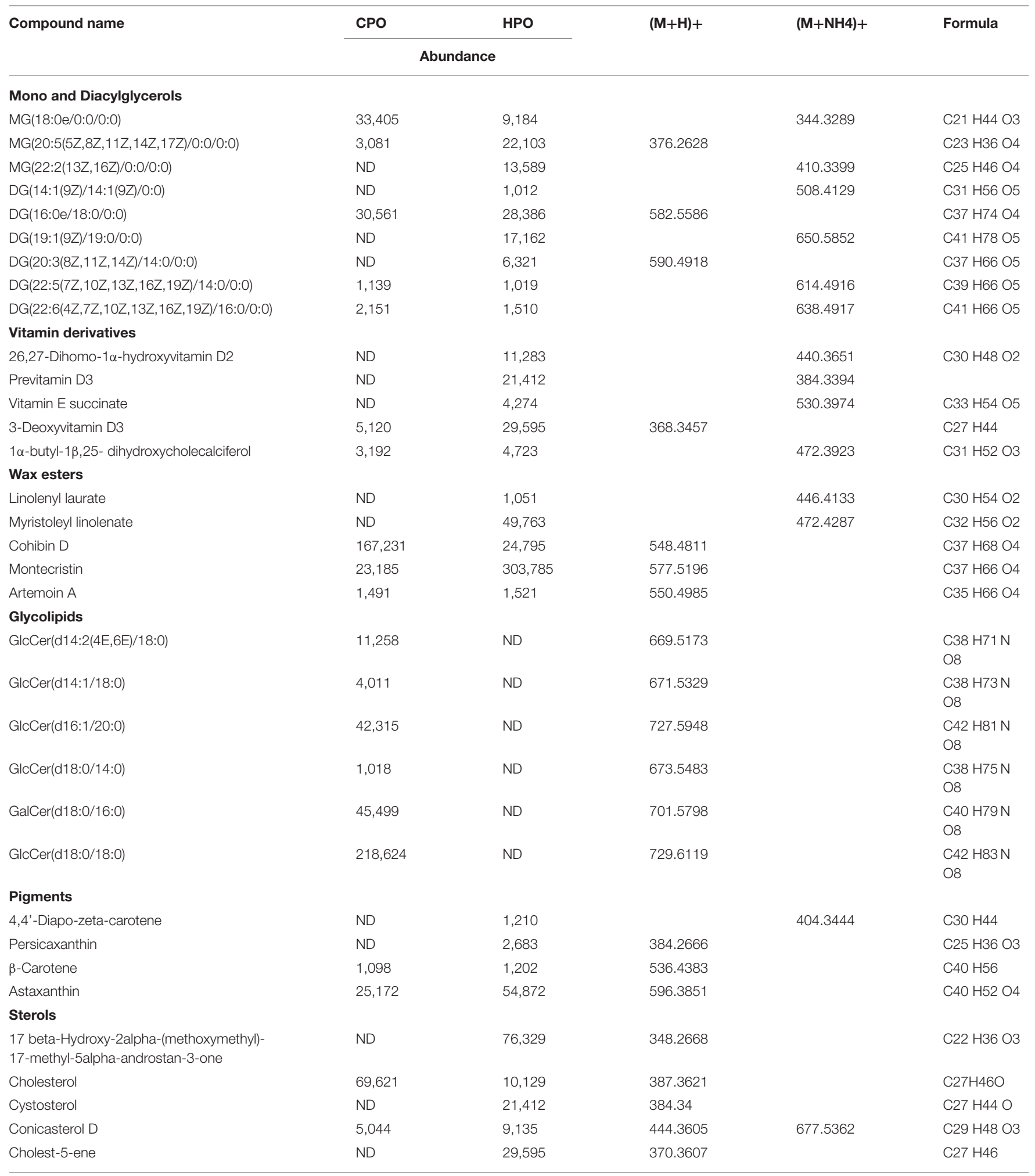


TABLE 2 | Continued

\begin{tabular}{|c|c|c|c|c|c|}
\hline Compound name & CPO & HPO & $(\mathrm{M}+\mathrm{H})+$ & $(\mathrm{M}+\mathrm{NH} 4)+$ & Formula \\
\hline 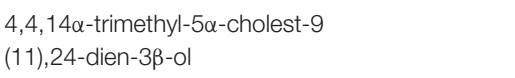 & 1,011 & ND & 426.3855 & & $\mathrm{C} 30 \mathrm{H} 50 \mathrm{O}$ \\
\hline $\begin{array}{l}4 \alpha \text {-methyl-24-methylene-cholestan- } \\
3 \beta, 8 \beta, 11 \beta \text {-triol }\end{array}$ & 13,100 & ND & 446.3757 & & $\mathrm{C} 29 \mathrm{H} 50 \mathrm{O} 3$ \\
\hline \multicolumn{6}{|l|}{ Phospholipids } \\
\hline PE(14:0/22:5(4Z,7Z,10Z,13Z,16Z)) & 1,129 & ND & 737.4995 & & $\begin{array}{l}\mathrm{C} 41 \mathrm{H} 72 \mathrm{~N} \\
\mathrm{O} 8 \mathrm{P}\end{array}$ \\
\hline PE(16:1(9Z)/P-18:1(11Z)) & 1,098 & 1,521 & 699.5207 & & $\begin{array}{l}\text { C39 H74 N } \\
\text { O7 P }\end{array}$ \\
\hline PE(16:0/22:6(4Z,7Z,10Z,13Z,16Z,19Z)) & 21,319 & ND & 763.516 & & $\begin{array}{l}\mathrm{C} 43 \mathrm{H} 74 \mathrm{~N} \\
\mathrm{O} 8 \mathrm{P}\end{array}$ \\
\hline $\mathrm{PE}(17: 1(9 Z) / 22: 6(4 Z, 7 Z, 10 Z, 13 Z, 16 Z, 19 Z))$ & 7,689 & ND & 775.514 & & $\begin{array}{l}\mathrm{C} 44 \mathrm{H} 74 \mathrm{~N} \\
\mathrm{O} 8 \mathrm{P}\end{array}$ \\
\hline PE(P-18:1(9Z)/18:4(6Z,9Z,12Z,15Z)) & 5,135 & ND & 721.5054 & & $\begin{array}{l}\mathrm{C} 41 \mathrm{H} 72 \mathrm{~N} \\
\mathrm{O} 7 \mathrm{P}\end{array}$ \\
\hline PE(P-18:1(9Z)/18:4(6Z,9Z,12Z,15Z)) & ND & 1,195 & 721.5073 & & $\begin{array}{l}\mathrm{C} 41 \mathrm{H} 72 \mathrm{~N} \\
\mathrm{O} 7 \mathrm{P}\end{array}$ \\
\hline PE(P-18:1(9Z)/20:5(5Z,8Z,11Z,14Z,17Z)) & ND & 1,152 & 747.5221 & & $\begin{array}{l}\mathrm{C} 43 \mathrm{H} 74 \mathrm{~N} \\
\mathrm{O} 7 \mathrm{P}\end{array}$ \\
\hline $\mathrm{PE}(18: 1(9 \mathrm{E}) / 18: 1(9 \mathrm{E}))$ & 14,047 & ND & & 743.5475 & $\begin{array}{l}\mathrm{C} 41 \mathrm{H} 78 \mathrm{~N} \\
\mathrm{O} 8 \mathrm{P}\end{array}$ \\
\hline $\begin{array}{l}\text { 1-Alkyl-2- } \\
\text { acylglycerophosphoethanolamine }\end{array}$ & 10,986 & 5,186 & 751.554 & & $\begin{array}{l}\mathrm{C} 43 \mathrm{H} 78 \mathrm{~N} \\
\mathrm{O} 7 \mathrm{P}\end{array}$ \\
\hline
\end{tabular}

LC-MS, Liquid chromatography-mass spectrometry; CPO, oil extracted from cephalothorax; HPO, oil extracted from hepatopancreas; ND, not detected; PE, Phosphatidylethanolamine; MG, Monoacylglycerol; DG, Diacylglycerol; GlcCer, Glycolipid.

used to monitor the formation of primary oxidation products, mainly hydroperoxides in the presence of $\mathrm{O}_{2}$. The formed hydroperoxides are not stable and more likely decomposed to secondary oxidation products such as aldehydes and ketones (3). PV was higher in CPO than HPO. For CDs, a higher CD was observed in HPO than CPO. CDs are also primary oxidation products. Lipid peroxidation starts with the abstraction of $\mathrm{H}$ from the $-\mathrm{CH}_{2}$ - group of PUFAs. As a result, carbon radical is stabilized by a molecular rearrangement, forming CDs in which, two double bonds are separated by a single bond (39). With a high content of PUFA, HPO could undergo a higher abstraction of $\mathrm{H}$, leading to the higher formation of CD. CDs were therefore defined as diene formation due to the presence of double bonds (21). However, the secondary oxidation products as indicated by TBARS value and AnV were higher in HPO. HPO was composed of higher PUFAs that were vulnerable to oxidation. At the beginning stage, primary oxidation products such as hydroperoxides were formed. Simultaneously, the decomposition occurred at a higher rate. As a result, the amount of hydroperoxide was decreased as indicated by lowered PV, while TBARS value and AnV were augmented. TBARS value has been widely used to measure volatile oxidation products, while AnV has been employed to measure non-volatile lipid oxidation products (3). AnV determines the amount of aldehydes, principally 2 -alkenals and 2, 4-dienals by reaction with p-anisidine (40). The results were in accordance with Gulzar and Benjakul (16) who found that the oxidation of shrimp oil from cephalothorax was enhanced during the storage period, while the 
addition of antioxidants could suppress the oxidation. Overall, $\mathrm{HPO}$ with high PUFA content was prone to oxidation than CPO containing a lower amount of PUFA (Table 1).

\section{FTIR Spectra}

Fourier transform infrared (FTIR) spectrum has been used for the identification of functional groups (41). Oil samples with the altered functional group formed due to oxidation could be identified via spectra (Figure 4). The wavenumber starting from 4,000 to $400 \mathrm{~cm}^{-1}$ was analyzed. The initial peak was found at 3,600-3,400 $\mathrm{cm}^{-1}$ in CPO and HPO, representing the $\mathrm{O}-\mathrm{OH}$ group (16). This denotes the presence of the primary oxidation product of peroxide, mainly hydroperoxide. However, the $\mathrm{O}-\mathrm{OH}$ peak was much lowered in HPO, representing the lower amount of peroxide formed. These results were in line with the PV result (Figure 3), in which higher PV was obtained in CPO than HPO. Other notable changes were found at 1,730-1,685 $\mathrm{cm}^{-1}$ and 1,200-970 $\mathrm{cm}^{-1}$, representing the unsaturated aldehydes (42) and P-OC (43), respectively. Similar peaks representing lipid oxidation in shrimp oil extracted by ultrasonic-assisted extraction were posted by Gulzar and Benjakul (44). The peak height of aldehydes was greater in HPO, compared to that of CPO, reflecting the augmented formation of secondary lipid oxidation products in HPO. Phosphate attached with the carbonyl group indicates the presence of higher phospholipids in CPO (6). However, the peak was lowered in HPO, indicating the lower phospholipid content. Overall, FTIR spectra revealed higher aldehydes, lower peroxide, and phospholipid in HPO than CPO.

\section{LC-MS Identification of Ethanol-Soluble Lipids}

Ethanol soluble lipids were prepared by the ethanol crystallization method (18). The ethanol-soluble polar and neutral lipids such as cholesterol, astaxanthin, fat-soluble vitamins, phospholipids, and other carotenoids were separated from insoluble non-polar lipids. The non-polar lipids mainly triglycerides were crystallized due to the insolubility in ethanol (6). In the current study, preliminary identification of lipid components in the ethanolic fraction was done by liquid chromatography-mass spectrometry (LC-MS) (Table 2). When comparing both the samples, CPO was dominated by glycolipids, phospholipids, diacylglycerol, monoacylglycerol, and sterol derivatives. In HPO, diacylglycerol, monoacylglycerol, and sterol derivatives were predominantly found. Although HPO contained phospholipids as one of the major components, some phospholipids were not identified, compared to those found in CPO. The result showed that the phospholipids in HPO might be lower, compared to that of CPO. FTIR results (Figure 4) supported the LC-MS data by showing the smaller peak of phospholipid in HPO. Glycolipids were dominant in CPO but they were not detected in HPO. Glycolipids and phospholipids are the major components in cell membrane regulating osmoregulatory changes in crustacean cell membrane structures (45). Cephalothorax contains more membranes or tissues, compared to hepatopancreas. The presence of lesser tissue in the hepatopancreas was due to the target removal of the gland by a sucking machine (26). This could be the reason for fewer phospholipids and unidentified glycolipids. Phospholipids in both oils mainly consisted of phosphatidylethanolamine (PE) conjugated with different fatty acids. In general, shrimp lipid contains $\mathrm{PE}$ and phosphatidylcholine as major phospholipids (13). However, the lipid components can vary due to seasonal changes, diet, maturity, and size (5). C30 column was used for the identification of astaxanthin esters and other neutral lipids in shrimp lipid (7), whereas C18 was the column used in the study. This could lead to the difference in profiles of components in oils. Other components found in both the samples were sterol derivatives, vitamin derivatives, $\alpha$-carotene, and astaxanthin. Based on the HPLC result, higher astaxanthin was found in HPO, thus providing more health benefits than CPO. Overall, the LC-MS identification revealed that HPO also contained vitamins with lesser phospholipid and higher astaxanthin contents.

\section{CONCLUSION}

Oil extracted from cephalothorax and hepatopancreas of Pacific white shrimp showed different compositions and nutrition indices. Oil from the hepatopancreas with higher yields had higher amounts of astaxanthin and lower cholesterol levels. PUFAs were more pronounced in HPO. Consequently, HPO possessed the superior nutritive value to CPO. However, secondary oxidation products were found in HPO, indicating that lipid oxidation took place. Nevertheless, the oxidation in both the samples could be overcome with the addition of natural antioxidants or exclusion of oxygen in the package.

\section{DATA AVAILABILITY STATEMENT}

The original contributions presented in the study are included in the article/supplementary material, further inquiries can be directed to the corresponding author/s.

\section{AUTHOR CONTRIBUTIONS}

NR and SG performed the research, analyzed the data, and wrote the original manuscript. NB provided technical support in data analysis. LM, XY, and BZ edited the manuscript. SB supervised the research design and reviewed the manuscript. All authors contributed to and approved the final draft of the manuscript.

\section{FUNDING}

This work was supported by Chair Professor Grant (P-20-52297). Thailand's Education Hub for Southern Region of ASEAN Countries (TEH-AC, 2018) scholarship and Prachayajarn Scholarship, Prince of Songkla University (Grant No. AGR6402088N) are also acknowledged. 


\section{REFERENCES}

1. Shiekh KA, Benjakul S, Gulzar S. Impact of pulsed electric field and vacuum impregnation with Chamuang leaf extract on quality changes in Pacific white shrimp packaged under modified atmosphere. LWT. (2021) 149:111899. doi: 10.1016/j.lwt.2021.111899

2. Mittal A, Singh A, Aluko RE, Benjakul S. Pacific white shrimp (Litopenaeus vannamei) shell chitosan and the conjugate with epigallocatechin gallate: antioxidative and antimicrobial activities. J Food Biochem. (2021) 45:e13569. doi: 10.1111/jfbc. 13569

3. Takeungwongtrakul S, Benjakul S, H-Kittikun A. Lipids from cephalothorax and hepatopancreas of Pacific white shrimp (Litopenaeus vannamei): compositions and deterioration as affected by iced storage. Food Chem. (2012) 134:2066-74. doi: 10.1016/j.foodchem.2012.04.003

4. Singh A, Mittal A, Benjakul S. Chitosan, chitooligosaccharides and their polyphenol conjugates: preparation, bioactivities, functionalities and applications in food systems. Food Hydrocoll. (2021) 6:1-23. doi: 10.1080/87559129.2021.1950176

5. Gulzar S, Raju N, Chandragiri Nagarajarao R, Benjakul S. Oil and pigments from shrimp processing by-products: extraction, composition, bioactivities and its application- a review. Trends Food Sci Technol. (2020) 100:30719. doi: 10.1016/j.tifs.2020.04.005

6. Raju N, Benjakul S. Application of saponin for cholesterol removal from Pacific white shrimp (Litopenaeus vannamei) lipid. Eur J Lipid Sci Technol. (2020) 122:2000078. doi: 10.1002/ejlt.202000078

7. Gómez-Estaca J, Calvo MM, Álvarez-Acero I, Montero P, Gómez-Guillén MC. Characterization and storage stability of astaxanthin esters, fatty acid profile and $\alpha$-tocopherol of lipid extract from shrimp (L. vannamei) waste with potential applications as food ingredient. Food Chem. (2017) 216:3744. doi: 10.1016/j.foodchem.2016.08.016

8. Ambati RR, Phang S-M, Ravi S, Aswathanarayana RG. Astaxanthin: sources, extraction, stability, biological activities and its commercial applications-a review. Mar Drugs. (2014) 12(Suppl):128-52. doi: 10.3390/md12010128

9. Nishino H, Murakosh M, Ii T, Takemura M, Kuchide M, Kanazawa M, et al. Carotenoids in cancer chemoprevention. Cancer Metastasis Rev. (2002) 21:257-64. doi: 10.1023/A:1021206826750

10. Wu H, Niu H, Shao A, Wu C, Dixon B, Zhang J, et al. Astaxanthin as a potential neuroprotective agent for neurological diseases. Mar Drugs. (2015) 13:5750-66. doi: $10.3390 / \mathrm{md} 13095750$

11. Navaneethan R, Vimaladevi S, Ajeesh Kumar KK, Anandan R, Niladri SC, Asha KK, et al. Profiling of Omega-3 polyunsaturated fatty acids of myctophid fish species available in Arabian sea. Fish Technol. (2016) 53:55-8. http://hdl. handle.net/123456789/2306

12. Sokoła-Wysoczańska E, Wysoczański T, Wagner J, Czyz K, Bodkowski $\mathrm{R}$, Lochyński $\mathrm{S}$, et al. Polyunsaturated fatty acids and their potential therapeutic role in cardiovascular system disorders-a review. Nutrients. (2018) 10:101561. doi: 10.3390/nu10101561

13. Miniadis-meimaroglou S, Sinanoglou VJ. Lipid profile and nutritional evaluation of shrimps. Lipidomics Sea Food, Mar Based Diet Suppl Fruit Seed. (2012) 661:21-40.

14. Chen J, Liu H. Nutritional indices for assessing fatty acids: a mini-review. Int J Mol Sci. (2020) 21:5695. doi: 10.3390/ijms21165695

15. Lusis AJ. Atherosclerosis. Nature. (2000) 407:233-41. doi: 10.1038/35025203

16. Gulzar S, Benjakul S. Effect of pre-treatments on yield and properties of lipid extracted from cephalothorax of Pacific white shrimp (Litopenaeus vannamei) by ultrasonic assisted process. LWT. (2019) 100:106-13. doi: 10.1016/j.lwt.2018.10.051

17. Raju N, Benjakul S. Use of beta cyclodextrin to remove cholesterol and increase astaxanthin content in shrimp oil. Eur J Lipid Sci Technol. (2019) 122:1900242. doi: 10.1002/ejlt.201900242

18. Raju N, Sae-leaw T, Osako K, Benjakul S. Improved cholesterol depletion with enhanced astaxanthin and polyunsaturated fatty acids of lipid from Pacific white shrimp cephalothorax using prior ethanolic separation of polar lipid and $\beta$-Cyclodextrin. J Food Sci Technol. (2021) 2021:110. doi: 10.1007/s13197-021-05238-7

19. Bavisetty SCB, Narayan B. An improved RP-HPLC method for simultaneous analyses of squalene and cholesterol especially in aquatic foods. J Food Sci Technol. (2015) 52:6083-9. doi: 10.1007/s13197-015-1793-4
20. Raju N, Singh A, Benjakul S. Recovery, reusability and stability studies of beta cyclodextrin used for cholesterol removal from shrimp lipid. RSC Adv. (2021) 11:23113-21. doi: 10.1039/D1RA03282H

21. Pudtikajorn K, Benjakul S. Simple wet rendering method for extraction of prime quality oil from skipjack tuna eyeballs. Eur J Lipid Sci Technol. (2020) 122:2000077. doi: 10.1002/ejlt.2020 00077

22. Firestone D. Official Methods and Recommended Practices of the AOCS. 7th ed. Champaign, IL: AOCS (2009).

23. Steel RGD, Torrie JH. Principles and Procedures of Statistics, a Biometrical Approach. 2nd ed. New York, NY: McGraw-Hill Kogakusha, Ltd. (1980).

24. Sachindra NM, Bhaskar N, Mahendrakar NS. Carotenoids in different body components of Indian shrimps. J Sci Food Agric. (2005) 85:16772. doi: $10.1002 /$ jsfa. 1977

25. Wang $\mathrm{W}, \mathrm{Wu} \mathrm{X}$, Liu Z, Zheng H, Cheng Y. Insights into hepatopancreatic functions for nutrition metabolism and ovarian development in the crab Portunus trituberculatus: gene discovery in the comparative transcriptome of different hepatopancreas stages. PLoS ONE. (2014) 9:84921. doi: 10.1371/journal.pone.0084921

26. Senphan T, Benjakul S, Kishimura H. Purification and characterization of trypsin from hepatopancreas of pacific white shrimp. J Food Biochem. (2015) 39:388-97. doi: $10.1111 /$ jfbc. 12147

27. Britton G. Functions of intact carotenoids. In: G Britton, S Liaaen-Jensen, H Pfander, editors, Carotenoids. Basel: Birkhäuser Basel. (2008). p. 189212. doi: 10.1007/978-3-7643-7499-0_10

28. Wade NM, Gabaudan J, Glencross BD. A review of carotenoid utilisation and function in crustacean aquaculture. Rev Aquac. (2017) 9:14156. doi: 10.1111/raq.12109

29. Crocke EL. Cholesterol function in plasma membranes from ectotherms: membrane-specific roles in adaptation to temperature. Am Zool. (1998) 38:291-304. doi: 10.1093/icb/38.2.291

30. Hobbs HH. Crustacea. Encycl Caves. (2012) 2012:17794. doi: 10.1016/B978-0-12-383832-2.00027-X

31. Kumar V, Sinha AK, Romano N, Allen KM, Bowman BA, Thompson $\mathrm{KR}$, et al. Metabolism and nutritive role of cholesterol in the growth, gonadal development, and reproduction of crustaceans. Rev Fish Sci Aquac. (2018) 26:254-73. doi: 10.1080/23308249.2018.1429 384

32. Shillito B, Desurmont C, Barthélémy D, Farabos D, Després G, Ravaux J, et al. Lipidome variations of deep-sea vent shrimps according to acclimation pressure: a homeoviscous response? Deep Sea Res I Oceanogr Res Pap. (2020) 161:103285. doi: 10.1016/j.dsr.2020.103285

33. Calder PC. The role of marine omega-3 (n-3) fatty acids in inflammatory processes, atherosclerosis and plaque stability. Mol Nutr Food Res. (2012) 56:1073-80. doi: 10.1002/mnfr.201100710

34. Ulbricht TLV, Southgate DAT. Coronary heart disease: seven dietary factors. Lancet. (1991) 338:985-92. doi: 10.1016/0140-6736(91)91846-M

35. Attia YA, Al-Harthi MA, Korish MA, Shiboob MM. Fatty acid and cholesterol profiles and hypocholesterolemic, atherogenic, and thrombogenic indices of table eggs in the retail market. Lipids Health Dis. (2015) 14:133. doi: 10.1186/s12944-015-0133-Z

36. Santos-Silva J, Bessa RJB, Santos-Silva F. Effect of genotype, feeding system and slaughter weight on the quality of light lambs: II. Fatty acid composition of meat. Livest Prod Sci. (2002) 77:187-94. doi: 10.1016/S0301-6226(02)00059-3

37. Simopoulos AP, Leaf A, Salem N. Essentiality of and recommended dietary intakes for omega-6 and omega-3 fatty acids. Ann Nutr Metab. (1999) 43:12730. doi: $10.1159 / 000012777$

38. Harika RK, Eilander A, Alssema M, Osendarp SJM, Zock PL. Intake of fatty acids in general populations worldwide does not meet dietary recommendations to prevent coronary heart disease: a systematic review of data from 40 countries. Ann Nutr Metab. (2013) 63:229-38. doi: 10.1159/000355437

39. Facundo HTF, Brandt CT, Owen JS, Lima VLM. Elevated levels of erythrocyte-conjugated dienes indicate increased lipid peroxidation in schistosomiasis mansoni patients. Brazilian J Med Biol Res. (2004) 37:95762. doi: 10.1590/S0100-879X2004000700003

40. Choe E, Min DB. Mechanisms and factors for edible oil oxidation. Compr Rev Food Sci Food Saf. (2006) 5:169-86. doi: 10.1111/j.1541-4337.2006.00009.x 
41. Singh A, Idowu AT, Benjakul S, Kishimura H, Aluko RE, Kumagai Y. Debittering of salmon (Salmo salar) frame protein hydrolysate using 2-butanol in combination with $\beta$-cyclodextrin: Impact on some physicochemical characteristics and antioxidant activities. Food Chem. (2020) 321:126686. doi: 10.1016/j.foodchem.2020.126686

42. Setiowaty G, Che Man YB, Jinap S, Moh MH. Quantitative determination of peroxide value in thermally oxidized palm olein by Fourier transform infrared spectroscopy. Phytochem Anal. (2000) 11:748. doi: 10.1002/(SICI)1099-1565(200003/04)11:2<74::AID-PCA498>3. $0 . \mathrm{CO} ; 2-\mathrm{E}$

43. Nzai JM, Proctor A. Determination of phospholipids in vegetable oil by fourier transform infrared spectroscopy. J Am Oil Chem Soc. (1998) 75:12819. doi: 10.1007/s11746-998-0173-x

44. Gulzar S, Benjakul S. Ultrasound waves increase the yield and carotenoid content of lipid extracted from cephalothorax of Pacific white shrimp (Litopenaeus vannamei). Eur J Lipid Sci Technol. (2018) 120:1700495. doi: 10.1002/ejlt.201700495

45. Huang M, Dong Y, Zhang Y, Chen Q, Xie J, Xu C, et al. Growth and lipidomic responses of juvenile pacific white shrimp Litopenaeus vannamei to low salinity. Front Physiol. (2019) 2019:1087. doi: 10.3389/fphys.2019.0 1087
Conflict of Interest: The authors declare that the research was conducted in the absence of any commercial or financial relationships that could be construed as a potential conflict of interest.

The handling editor declared a past co-authorship with one of the authors, SB.

Publisher's Note: All claims expressed in this article are solely those of the authors and do not necessarily represent those of their affiliated organizations, or those of the publisher, the editors and the reviewers. Any product that may be evaluated in this article, or claim that may be made by its manufacturer, is not guaranteed or endorsed by the publisher.

Copyright (C 2021 Raju, Gulzar, Buamard, Ma, Ying, Zhang and Benjakul. This is an open-access article distributed under the terms of the Creative Commons Attribution License (CC BY). The use, distribution or reproduction in other forums is permitted, provided the original author(s) and the copyright owner(s) are credited and that the original publication in this journal is cited, in accordance with accepted academic practice. No use, distribution or reproduction is permitted which does not comply with these terms. 